\title{
Matrix Approach to Project Planning Design and Management
}

\author{
B.A Oseni ${ }^{1}$, T.A Lasisi ${ }^{2}$, A. Rahaman ${ }^{3}$ \\ ${ }^{1,2}$ Department of Mathematics and Statistics. The Polytechnic, Ibadan. \\ ${ }^{3}$ Department of Science Laboratory Technology.The Polytechnic, Ibadan
}

\begin{abstract}
The failure of many projects, be it, Federal, states or local government is due in many cases, to poor design. Design can be defined as the formal framework that establishes, in advance, what the intention of the project is; a logical plan of activities, with the means to measure progress, taking into consideration the environmental factors and other socio-political factors that could affect the assumptions on which the project is based. The matrix method is primarily a device of project planning which define the project inputs, outputs and the intermediate and higher objectives in measurable terms.

Targets are established to permit subsequent measurement or verification of achievement of the defined outputs and objective. The paper proposes a methodology to the design of a successful project and an example of the matrix method as applied to a development project.
\end{abstract}

\section{Preamble:}

The failure of many projects is due in most cases to poor design. The design is the formal Framework that establishes the intention of the project. It is a Logical plan of activities, with the means to measure progress, taking into consideration the environmental factors and socio political factors that could affect the assumptions on which the project is based.

An integral part of project planning is both the design and evaluation, evaluation showing the result of comparing plans with the actual accomplishment.

Several projects, are bases on the design shown in figure 1 below which is limited in its effectiveness.

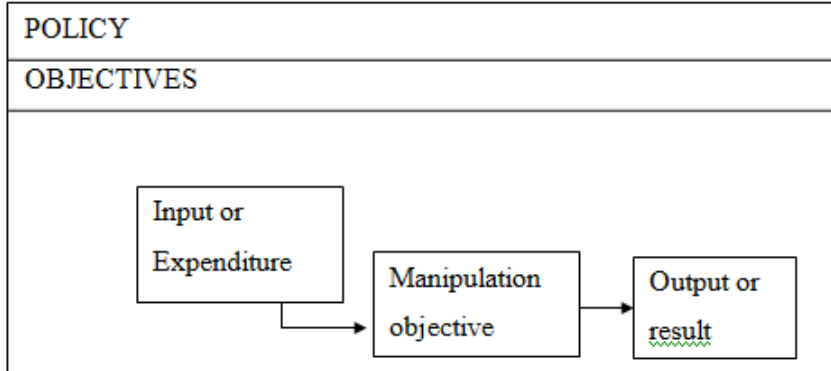

Figure 1: Typical Project design.

It should be noted that, it. is insufficient to treat the design as a set of imputes activities and outputs with some policy statement and one objective.

The first stage of any design is to identify the problem to be solved and its causes before solving the problem. At the appraisal and preparation stage, there should be a consistency between the donor's policy objectives and the recipient's needs. The design should commence with a project document, which provides a solid foundation for subsequent implementation.

To start with, there should be a statement presenting the problem or situation under study, briefly at this stage, for which a solution is sought.

This stage should not attempt to suggest means or procedures of solving the problem and whether the proposed project is justified, these will be examined in the design.

As a matter of fact, a service of objectives needs to be set so that answers to the following questions can be given why is the project being designed? What are the reasons for the project? What is the project expected to solve if completed successfully and to time? And finally, the question of who will benefit from the project?

There should be several levels of objectives depending on the type of project under consideration and to be specified, a definition of the target group. This target group definition should state who will be the beneficiaries if the project is completed on time. In many instances, a governmental agency or organization will receive direct assistance and then through them, the target group will benefit. 


\section{Objectives}

An objective is a simple expression of a deceived end. In formulating an objective, the designer should not confuse ends with means. At the objective level, one is not so much interested in the approaches being used, as these are described under output and activities, as in the effect the project is expected to have.

In general rule, the project should be formulated in terms of a single National, States or Local objective and a single intermediate objective.

\section{National, States Or Local Objecitves.}

Depending on which one of the three levels in appropriate, the National, State or Local objective should give the reason for the project, the broader objective towards which the efforts of the project is directed. Once the objective level has been chosen and formulated, it should be verified that

(a) It gives the reason for the project, which is the level beyond the intermediate objective.

(b) It states a single objective or a group of compatible objectives; it does not contain two or more objectives that are on different levels and,

(c) It is so presented that progress towards it can be assessed.

For instance, the modernization of the Nigeria agricultural sector, and increased yield per fed and is intended to result in expended exports of agricultural crops.

\section{Intermediate Objective}

The intermediate objective gives the primary reason for the project, that is the development that is expected to be achieved or the problem to be solved if the project is completed successfully to time.

However, the statement of the intermediate objective should name the group to whom the project is directed and the effect sought on completion of the project.

Hence, once the intermediate objective has been formulated, it should be verified that.

- The statement specifies what effect will have been achieved if the

project is successfully completed on time.

- A single intermediate objective preferably is stated,

- The intermediate objective is stated in terms of ends, not of means.

For instance, by the year 2000, the Nigeria Agricultural Universities will be capable of graduating 250 qualified agricultural specialists annually without any external/foreign assistance, and an irritation network scheme in the Nigerian Ministry of agricultural project is intended to produce increased per fed and yield.

Furthermore, there could be more objectives at the local or State level, which describe objectives beyond the intermediate stage.

\section{Inputs, Outputs And Activities.}

The inputs are the means, including money, equipment, materials, technical advice, training, supervision etc. which are later mobilized to produce the planned outputs while the activities are where time and resources are used to transform the inputs into outputs. At this juncture, specific questions can be answered such as,

- What will the machine do'?

- What will the staff actually do?

The outputs are the specifically intended results, as opposed to the magnitude of results that can be expected from good management of the inputs provided.

For example, manpower, machinery and training (inputs) allow the training of staff, ordering of equipment and materials (activities), for trained operational staff with material and equipment in a specific place (outputs) when managed efficiently to produce an irritation network at this stage of the design, the system shown in figure 2 will exit.

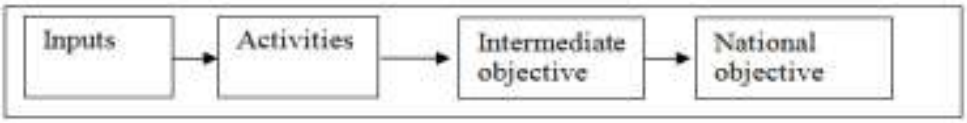

Figure2: Design sequence.

Clarification of this relationships or linkage is the basis of the design concept.

\section{Project Linkages.}

There is causative link between project outputs, such as (the irritation network)/(trained operational staff) and the intermediate project objective, such as (improved crop yields) / qualified agricultural specialists).

The outputs must be made available before the objective can be achieved. The presence of the output does not, however, guarantee that the objective will be reached. Environmental factors like farmer attitudes, lack 
of credit facilities, political interference, may prevent the objective achieved. The causative relationship between the project outputs and the intermediate project objective must be carefully stated and thus, used as a hypothesis under testing, with the environmental factors being identified. The evaluation will then conclude whether or not the hypothesis was realized.

There is also a causative link between the intermediate objective, (improved

crop yields)/(qualified agricultural specialists), and progress towards the National objective, (expended export of agricultural crop) / (modernization of Nigeria's agricultural sector).

The achievement of the intermediate project objective does not guarantee that the National objective will be reached. Environmental factors could again affect planned progress, such as (price and marketing conditions) / (emigration of trained staff), towards the highest level objectives. Thus, the causative relationship between the intermediate objective and the National objective must be clearly stated as a hypothesis with the environmental factors identifies and the hypothesis validated (see figure 3 and 4)

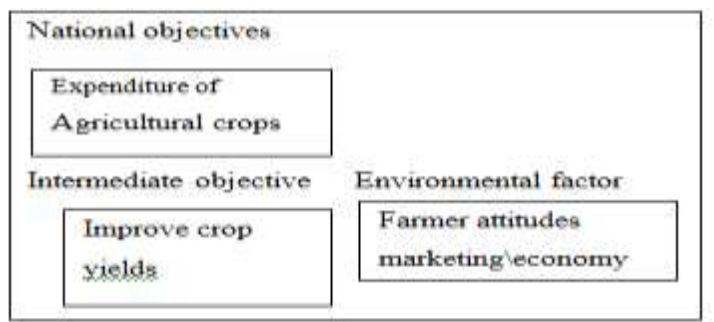

Figure 3: Example of relationship between objectives.

The number of levels between inputs and the ultimate objective may be greater than five shown in figure 4 . What is paramount is the train of though linking one level to the next. Thus, the project planner may find if necessary to expend the number of levels to adequately reflect the realities of the particular project.

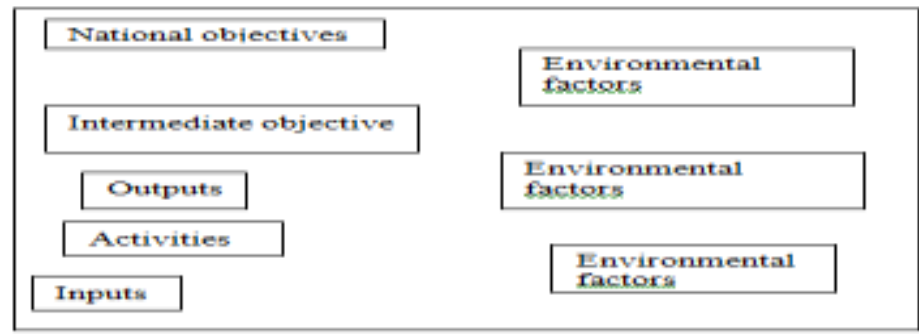

Figure 4. The levels between input and ultimate objective.

Further objective levels, such as local and State, may be appropriate as well as the separating of outputs or activities into two or more distinct phases.

\section{Tarcer Setting}

There are a number of problems associated with target setting and quantification. Many projects set targets at the output, intermediate and higher objective levels only, but if the project is broken down into distinct phases, then targets at the input stage through to ultimate objective can be extremely effective.

An obvious problem is that, in many cases, it will only be possible to quanti part of an objective and because only His part of the project is able to be checked, the remainder many suffer from lack of attention.

In the example of training agricultural specialists, the target 250 may of the particular project. National objectives become more important than quality of those graduating. Yet a qualitative target could be incorporated in the project, such as the number of contact hours for specify subjects accepted internationally as the norm for this discipline. What is important is that measures or indicators will demonstrate at each level that the set targets are being achieved and that the project is effective and having the desired impact on the target group.

\section{Monitoring And Evaluation.}

An important benefit of setting targets at the various levels is that it focuses the attention of the project analyst on the data requirements for monitoring and evaluation. It may become obvious that a baseline survey will be necessary, particularly when people centered projects are being designed.

Acknowledge of statistical sampling becomes a great advantage at this stage; knowing what size of sample will incur a particular sampling error can avoid a very time consuming and expensive survey. The advice 
of a statistician should be sought if knowledge of surveys and sampling is limited. The important point is that a well-designed project should be able to highlight the need for a data collecting system.

A well-designed project should incorporate those factors, objectives and systems as indicated in figure 5.

Environmental factors

National objectives

\begin{tabular}{|l|l|}
\hline \multicolumn{2}{|l|}{ State objectives } \\
\hline Local objectives \\
\hline Intermediate objectives \\
\hline Monitor measurement \\
levaluate \\
\begin{tabular}{|l|l|l|l|}
\hline Tnput & Targets \\
\hline
\end{tabular} \\
\hline
\end{tabular}

Figure 5. Proposed project design

\section{Matrix Methodology}

A key element in project planning and evaluation is the working part of a logical matrix, which:

- Defines project inputs, output, intermediate and higher objectives in measurable or objectively verifiable terms.

- Hypothesizes the causative (means-end) linkage between inputs, outputs, intermediate and higher objectives,

- Articulates environmental factors, which will affect the causative linkages

- Establishes the targets, which will permit subsequent measurement or verification of achievements of the defined outputs and objectives.

The matrix is primarily a project planning device it is also used for re examination of the original design of on going projects, as a necessary prelude to evaluation (it sets the standards against which the project will be evaluation). Evaluation then consists of determining and validating whether or not the project outputs are being produced; whether these outputs are serving to achieve the project purpose; and whether this achievement in making a significant contribution, as planned to the higher objectives.

The matrix has both vertical and horizontal logic. Its vertical logic defines the series of causative linkages, which is intended to transform project inputs into development changes at the various levels, and permits the planner / evaluator to judge whether these linkages are viable. Its horizontal logic encourages the evaluator to measure progress and verify planning assumptions at each level (inputs, activities, outputs, objectives and targets) separately and independently from other level.

\section{Characteristics And Limitation Of The MatrixMethod}

- The matrix method is ethically neutral.

- The matrix method is programmatically and technologically neutral.

- The methodology permits, but does require cost / benefit and cost / effectiveness analysis.

- It introduces order and discipline into the intellectual processes of the planner.

- The matrix method is merely a convenient planner's tool, which stimulates and visually displays the project designs so that they can be manipulated, assayed and communicated.

- The matrix method is objective-oriented, it does not describe the actions, activities or processes which transform means into ends.

\section{References}

[1] ANON (1984), Design and Evaluation for UNIDO Executed Projects and Programmes UNIDO USA.

[2] ANON (1981), Guidelines on Logical Framework Planning and Project Benefit Monitoring Evaluation Agricultural and Rural Development Asian Development Bank, Indonesia.

[3] CASLEY, D.J and LURRY, D.A (1982), Monitoring and Evaluation of Agricultural Development IBRD, UK.

[4] CHAMBERS, R: (1980), Repaid Rural Appraisal, IDS Discussion Paper No 155, UK. 